San Jose State University

SJSU ScholarWorks

Faculty Research, Scholarly, and Creative Activity

$11-20-2020$

\title{
Non-Hermitian topology of one-dimensional spin-torque oscillator arrays
}

Benedetta Flebus

University of Texas at Austin

Rembert A. Duine

Utrecht University

Hilary M. Hurst

San Jose State University, hilary.hurst@sjsu.edu

Follow this and additional works at: https://scholarworks.sjsu.edu/faculty_rsca

Part of the Condensed Matter Physics Commons

\section{Recommended Citation}

Benedetta Flebus, Rembert A. Duine, and Hilary M. Hurst. "Non-Hermitian topology of one-dimensional spin-torque oscillator arrays" Physical Review B (2020). https://doi.org/10.1103/PhysRevB.102.180408

This Article is brought to you for free and open access by SJSU ScholarWorks. It has been accepted for inclusion in Faculty Research, Scholarly, and Creative Activity by an authorized administrator of SJSU ScholarWorks. For more information, please contact scholarworks@sjsu.edu. 


\title{
Non-Hermitian topology of one-dimensional spin-torque oscillator arrays
}

\author{
Benedetta Flebus, ${ }^{1}$ Rembert A. Duine $\odot,{ }^{2,3,4}$ and Hilary M. Hurst $\odot^{5,6}$ \\ ${ }^{1}$ Department of Physics, The University of Texas at Austin, Austin, Texas 78712, USA \\ ${ }^{2}$ Institute for Theoretical Physics and Center for Extreme Matter and Emergent Phenomena, Utrecht University, \\ Leuvenlaan 4, NL-3584 CE Utrecht, The Netherlands \\ ${ }^{3}$ Department of Applied Physics, Eindhoven University of Technology, P.O. Box 513, NL-5600 MB Eindhoven, The Netherlands \\ ${ }^{4}$ Center for Quantum Spintronics, Department of Physics, Norwegian University of Science and Technology, NO-7491 Trondheim, Norway \\ ${ }^{5}$ Joint Quantum Institute, National Institute of Standards and Technology, and University of Maryland, Gaithersburg, Maryland 20899, USA \\ ${ }^{6}$ Department of Physics and Astronomy, San Jose State University, San Jose, California 95192, USA
}

(Received 2 March 2020; accepted 6 November 2020; published 20 November 2020)

\begin{abstract}
Magnetic systems have been extensively studied from both a fundamental physics perspective and as technological building blocks. The topological properties of magnonic excitations in these systems remain relatively unexplored, due to their inherently dissipative nature. The recent extension of the theory of topological classification to non-Hermitian Hamiltonians provides a pathway to engineer topological phases in dissipative systems. Here, we propose a magnonic realization of a topological, non-Hermitian system. A crucial ingredient of our proposal is the injection of spin current into the magnetic system, which alters and can even change the sign of terms describing dissipation. We show that the magnetic dynamics of an array of spin-torque oscillators can be mapped onto a non-Hermitian Su-Schrieffer-Heeger model exhibiting topologically protected edge states. The nontrivial topological phase is accessed by tuning the spin current injected into the array. We derive this result using both exact diagonalization of the effective non-Hermitian Hamiltonian and numerical analysis of the nonlinear equations of motion. In the nontrivial topological phase, a single spin-torque oscillator on the edge of the array is driven into auto-oscillation and emits a microwave signal, while the bulk oscillators remain inactive. Our findings have practical utility for memory devices and spintronics neural networks relying on spin-torque oscillators as constituent units.
\end{abstract}

DOI: 10.1103/PhysRevB.102.180408

Introduction. Topology has been a cornerstone in understanding condensed matter systems since the discovery of the quantum Hall effect in 1980 [1]. Connecting concepts drawn from topology to electronic systems led to the understanding of physical phenomena such as the quantum anomalous Hall [2] and quantum spin Hall effects [3], and the discovery of new materials including Weyl semimetals [4], topological superconductors [5], and topological insulators [6]. One fascinating aspect of topology is the bulk-boundary correspondence [7], in which bulk system properties predict the existence of localized boundary modes between topologically distinct systems. These modes are topologically protected, meaning they are robust with respect to environmental perturbations, and have thus been proposed as platforms for diverse applications from electric circuits to quantum computation [8]. Topological phases of matter have also been realized beyond electronic systems, for example, in ultracold atoms [9], photonic crystals [10], and mechanical systems [11].

The topological properties of magnons, i.e., linear excitations of magnetic systems, have been considered [12]. However, this direction of research remains relatively unexplored, in part because magnonic systems are inherently dissipative. The magnon number is not conserved due to ubiquitous spin nonconserving interactions with the crystalline lattice [13]. The conventional classification of topological properties relies on a Hermitian description, restricting the theory to closed systems without dissipation.

(a)

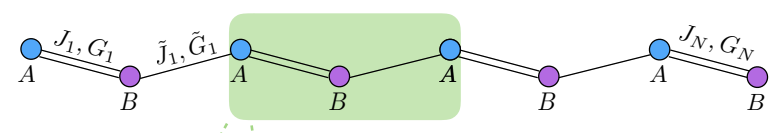

(b)

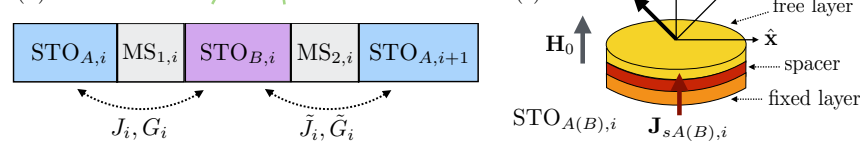

FIG. 1. Schematic illustration of our model. (a) A spin-torque oscillator (STO) array represented as a one-dimensional lattice with sublattices $A$ and $B$. Nearest-neighbor hopping in the $i$ th unit cell (with $i=1, \ldots, N$ ) is denoted $J_{i}, G_{i}$ and $\tilde{J}_{i}, \tilde{G}_{i}$ for intra- and intercell hopping, respectively. (b) Each unit cell consists of two STOs, labeled $\mathrm{STO}_{A, i}$ and $\mathrm{STO}_{B, i}$, and two metallic spacers connecting adjacent STOs, denoted $\mathrm{MS}_{1(2), i}$. Metallic spacers $\mathrm{MS}_{1(2), i}$ mediate both a reactive RKKY-like coupling and a dissipative spin-pumping coupling between spins of $\mathrm{STO}_{A, i}\left(\mathrm{STO}_{B, i}\right)$ and $\mathrm{STO}_{B, i}\left(\mathrm{STO}_{A, i+1}\right)$, whose strength is parametrized, respectively, by $J_{i}\left(\tilde{J}_{i}\right)$ and $G_{i}\left(\tilde{G}_{i}\right)$. (c) Each STO is a free ferromagnet|spacer|fixed ferromagnet trilayer subjected to the spin current $\mathbf{J}_{s A(B), i}$. An external magnetic field $\mathbf{H}_{0}$ sets the equilibrium direction of the magnetic order parameter $\mathbf{m}_{A(B), i}$ of the free layer. Other implementations of STOs are possible. 
The recent introduction of topological classifications for non-Hermitian Hamiltonians opens up new prospects for realizing nontrivial topological phases in intrinsically dissipative systems $[14,15]$. While a complete theoretical background it is still missing, recent experimental developments have shown that topologically protected edge modes can appear in dissipative systems under the right conditions [16].

In this Rapid Communication, we propose a nonHermitian, topological magnonic system. As illustrated in Fig. 1, we consider a one-dimensional (1D) array of spintorque oscillators (STOs) [17], which are current-driven magnetic nanopillars. In the regime of linear dynamics, this array is a magnonic realization of a non-Hermitian Su-SchriefferHeeger $(\mathrm{SSH})$ model with parity-time $(\mathcal{P} \mathcal{T})$ symmetry or chiral-inversion (CI) symmetry [18-20]. Different symmetry classes are accessed by changing experimentally controllable parameters. The injection of spin current into the magnets is a crucial ingredient of our setup, because it counteracts and can even overcome damping. Using exact diagonalization and a numerical solution of the nonlinear equations describing the magnetization dynamics, we show that this system displays a robust topological phase where a single STO at the edge is driven into auto-oscillation while the bulk remains inactive.

Model. We consider a one-dimensional array of $2 N$ STOs arranged in $N$ unit cells, as shown in Fig. 1. The $i$ th unit cell consists of two STOs, labeled $\mathrm{STO}_{A, i}$ and $\mathrm{STO}_{B, i}$, and two metallic spacers coupling the intracell and intercell STO elements, denoted $\mathrm{MS}_{1, i}$ and $\mathrm{MS}_{2, i}$, respectively. The dynamics of the magnetic order parameter $\mathbf{m}_{\eta, i}$ (with $\eta=A, B$ ) of an isolated spin-torque oscillator $\mathrm{STO}_{\eta, i}$ subject to a magnetic field $\mathbf{H}_{0}=H_{0} \hat{\mathbf{z}}$ and a spin current (in units of frequency) $\mathbf{J}_{s \eta, i}=J_{s \eta, i} \hat{\mathbf{z}}$ is [21]

$$
\begin{aligned}
\left.\dot{\mathbf{m}}_{\eta, i}\right|_{0}= & \omega_{\eta, i} \hat{\mathbf{z}} \times \mathbf{m}_{\eta, i}+\alpha_{\eta, i} \mathbf{m}_{\eta, i} \times \dot{\mathbf{m}}_{\eta, i} \\
& +J_{s \eta, i} \mathbf{m}_{\eta, i} \times\left(\mathbf{m}_{\eta, i} \times \hat{\mathbf{z}}\right) .
\end{aligned}
$$

Here, $\omega_{\eta, i}=\gamma_{\eta, i}\left(H_{0}-4 \pi M_{\eta, i}\right)$ is the ferromagnetic resonance frequency, with $\gamma_{\eta, i}$ the gyromagnetic ratio, $M_{\eta, i}$ the saturation magnetization, and $\alpha_{\eta, i} \ll 1$ is the (dimensionless) Gilbert damping parameter. The second and third terms on the right-hand side of Eq. (1) are, respectively, the dissipative torque accounting for energy dissipation [22] and the Slonczewski-Berger spin-transfer torque describing the interaction of the magnetic order parameter with the spin-polarized current [23]. In our proposed setup, the spin-transfer torque acts as a dissipative process that counteracts the intrinsic dissipation and thus provides tunable gain. Metallic spacers can mediate both reactive and dissipative coupling between STOs. For the reactive coupling, we consider a RudermanKittel-Kasuya-Yosida (RKKY)-type exchange whose strength is parametrized by the frequency $J_{i}\left(\tilde{J}_{i}\right)$ for the spacer $\mathrm{MS}_{1, i}\left(\mathrm{MS}_{2, i}\right)$. In our convention, $J_{i}\left(\tilde{J}_{i}\right)>0$ corresponds to a ferromagnetic exchange coupling. The dissipative coupling is mediated by spin pumping through the spacer $\mathrm{MS}_{1, i}$ $\left(\mathrm{MS}_{2, i}\right)$ [24]; its efficiency is parametrized by the dimensionless parameter $G_{i}\left(\tilde{G}_{i}\right) \ll 1$, which is microscopically related to the spin-mixing conductance of the oscillator|metallic spacer interface [25]. Note that we ignore dipolar interactions. Such interactions are made small by considering synthetic antiferromagnets as the free layers of the spin-torque oscillators [26]. Assuming a nearest-neighbor coupling, the coupled dynamics introduced via the metallic spacers reads as

$$
\begin{aligned}
\left.\dot{\mathbf{m}}_{A, i}\right|_{\text {coup }}= & -\mathbf{m}_{A, i} \times\left(J_{i} \mathbf{m}_{B, i}+\tilde{J}_{i-1} \mathbf{m}_{B, i-1}\right) \\
& +G_{i}\left[\mathbf{m}_{A, i} \times \dot{\mathbf{m}}_{A, i}-\mathbf{m}_{B, i} \times \dot{\mathbf{m}}_{B, i}\right] \\
& +\tilde{G}_{i-1}\left[\mathbf{m}_{A, i} \times \dot{\mathbf{m}}_{A, i}-\mathbf{m}_{B, i-1} \times \dot{\mathbf{m}}_{B, i-1}\right], \\
\left.\dot{\mathbf{m}}_{B, i}\right|_{\text {coup }}= & -\mathbf{m}_{B, i} \times\left(J_{i} \mathbf{m}_{A, i}+\tilde{J}_{i} \mathbf{m}_{A, i+1}\right) \\
& +G_{i}\left[\mathbf{m}_{B, i} \times \dot{\mathbf{m}}_{B, i}-\mathbf{m}_{A, i} \times \dot{\mathbf{m}}_{A, i}\right] \\
& +\tilde{G}_{i}\left[\mathbf{m}_{B, i} \times \dot{\mathbf{m}}_{B, i}-\mathbf{m}_{A, i+1} \times \dot{\mathbf{m}}_{A, i+1}\right],
\end{aligned}
$$

where $\tilde{G}_{1(N)}=\tilde{J}_{1(N)}=0$. The sum of Eqs. (1) and (2) determines the full system dynamics. In the following, we consider identical unit cells and drop the parameter dependence on index $i$. We assume that the magnetic field is large enough to order the magnets along its direction, and proceed to linearize Eqs. (1) and (2) around the equilibrium direction of the magnetic order parameter. That is, we write $\mathbf{m}_{\eta, i}=\left(m_{\eta, i x}, m_{\eta, i y}, 1\right)$, with $\left|\mathbf{m}_{\eta, i}\right| \simeq 1$. Next, we introduce the complex variable $2 m_{\eta, i}^{-}=m_{\eta, i x}-i m_{\eta, i y}$ and invoke the Holstein-Primakoff transformation [27] $m_{A(B), i}^{-}(t)=$ $\left\langle a_{i}\left(b_{i}\right)\right\rangle e^{-i \omega t}$, where the second-quantized operator $a_{i}\left(b_{i}\right)$ annihilates a magnon at the sublattice $A(B)$ of the $i$ th unit cell and obeys bosonic commutation relations.

Assuming the local damping to be much larger than the nonlocal one, $\tilde{G}, G \ll \alpha_{\eta}$, the linearized dynamics that follows from Eqs. (1) and (2) corresponds to a non-Hermitian SSH model with complex on-site potentials and complex intercell and intracell couplings. For identical STOs on the $A$ and $B$ sublattices we have $\omega_{\eta} \equiv \omega$ and $\alpha_{\eta} \equiv \alpha$. The corresponding Hamiltonian is $H=\sum_{i=1}^{N} H_{i}$, with $H_{i}$ the Hamiltonian for the $i$ th unit cell, which reads

$$
\begin{aligned}
H_{i}= & \omega\left[a_{i}^{\dagger} a_{i}+b_{i}^{\dagger} b_{i}\right]+i\left(J_{s A}-\alpha \omega\right) a_{i}^{\dagger} a_{i} \\
& +i\left(J_{s B}-\alpha \omega\right) b_{i}^{\dagger} b_{i}+(-J+i G \omega)\left[a_{i}^{\dagger} b_{i}+\text { H.c. }\right] \\
& +(-\tilde{J}+i \tilde{G} \omega)\left[a_{i}^{\dagger} b_{i-1}+\text { H.c. }\right],
\end{aligned}
$$

for $i \neq 1, N$, with open boundary conditions

$$
\begin{aligned}
H_{j}= & \omega a_{j}^{\dagger} a_{j}+\omega b_{j}^{\dagger} b_{j}+i\left(J_{s A}-\alpha \omega\right) a_{j}^{\dagger} a_{j} \\
& +i\left(J_{s B}-\alpha \omega\right) b_{j}^{\dagger} b_{j}+(-J+i G \omega)\left[a_{j}^{\dagger} b_{j}+b_{j}^{\dagger} a_{j}\right] \\
& +(-\tilde{J}+i \tilde{G} \omega) b_{j}^{\dagger} a_{l},
\end{aligned}
$$

with $j=1, N$ and $l=2, N-1$. Equations (3) and (4) constitute our starting point for exact diagonalization.

Results. The effective Hamiltonian in Eqs. (3) and (4) is a realization of a non-Hermitian SSH chain with many tunable features affecting its symmetries. We investigate the topology as a function of $J / \tilde{J}$, which can be experimentally controlled by tuning the length of the metallic spacers. Here, we consider the case where spin current is injected only at the $A$ sites, setting $J_{s A}=J_{s}$ and $J_{s B}=0$. Importantly, $H$ does not exhibit the non-Hermitian skin effect. Therefore an analysis of its topology based on periodic boundary conditions correctly indicates the presence or absence of topologically protected edge modes for the open system [14,20,28,29]. For systems exhibiting the non-Hermitian skin effect this is no longer true. 

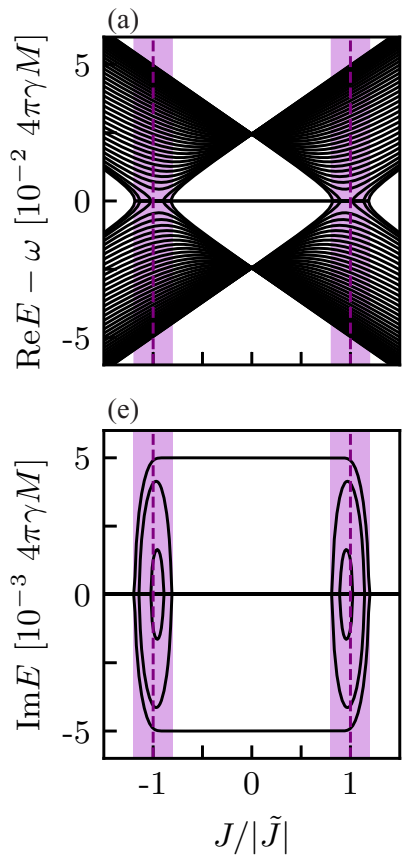

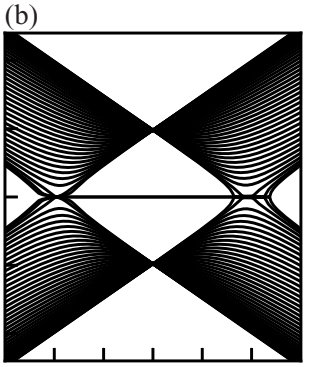

(f)

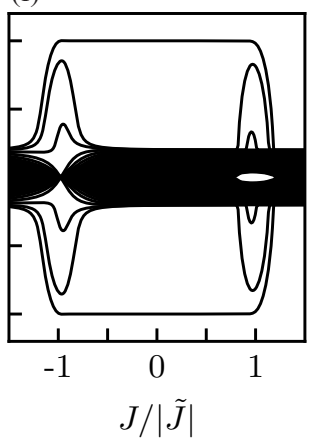

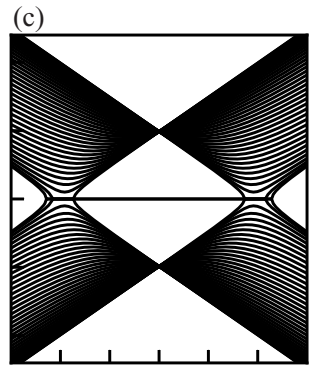

(g)

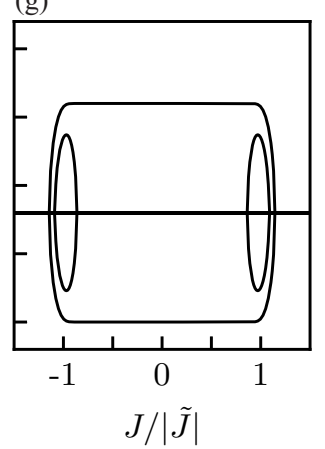

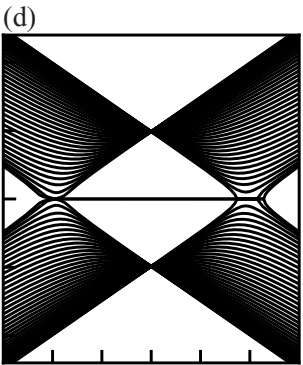

(h)

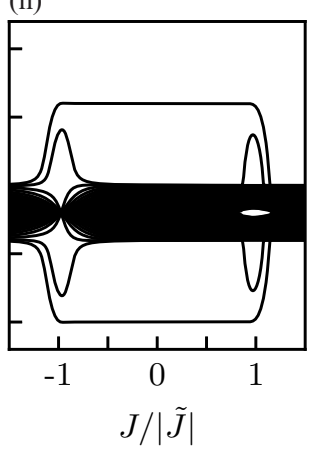

FIG. 2. Exact diagonalization of Eqs. (3) and (4) for $N=40$ unit cells (80 STOs) with $\omega / 4 \pi \gamma M=0.5, \alpha=0.01, \tilde{J} / 4 \pi \gamma M=0.025$. The columns correspond to the four symmetry regimes of $H_{k}$ discussed in the main text. (a)-(d) show $\operatorname{Re} E-\omega$ and (e)-(h) Im $E$. All cases have a line gap on the real axis and two edge modes with $\operatorname{Re} E-\omega=0$ for $J \lesssim|\tilde{J}|$. The real part of the spectrum is mostly unaltered in each case, aside from small deviations of the gap closing point. (a), (e) $\mathcal{P} \mathcal{T}$-symmetric case. There is one lasing edge mode for $J<|\tilde{J}-\alpha \omega|$, whereas

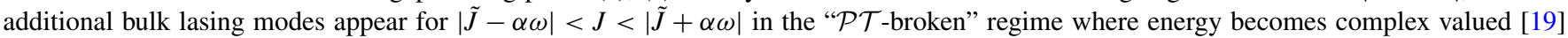
(purple/light gray shaded area). The topological transition occurs at $J= \pm \tilde{J}$ (purple/gray dashed line). (b), (f) Nonzero dissipative couplings $G, \tilde{G}=0.1 \alpha$. Bulk modes have $\operatorname{Im} E \neq 0$, but there is still a well-defined lasing edge mode separated from the bulk modes for $J \lesssim|\tilde{J}|$. This regime has chiral-inversion (CI) symmetry. (c), (g) Deviation of $J_{s}$ from the $\mathcal{P} \mathcal{T}$-symmetric value $J_{s}^{\mathrm{PT}}=2 \alpha \omega$ where $J_{s}=0.8 J_{s}^{\mathrm{PT}}$. For $J_{s}<J_{s}^{\mathrm{PT}}$ the imaginary spectrum is shifted down, while for $J_{s}>J_{s}^{\mathrm{PT}}$ it is shifted up (not shown). The edge mode is still lasing (Im $E>0$ ) for $J_{s} \gtrsim \alpha \omega$. This regime has chiral symmetry (CS). (d), (h) Combination of the two $\mathcal{P} \mathcal{T}$-breaking terms, $G, \tilde{G}=0.1 \alpha$ and $J_{s}=0.8 J_{s}^{\mathrm{PT}}$. Im $E$ has properties of (f) and (g). Both CI and CS are broken, but TRS ${ }^{\dagger}$ symmetry is preserved.

However, it is not straightforward to modify our model such that it exhibits the non-Hermitian skin effect, while being still experimentally relevant; thus, we leave such generalizations to future work.

The Fourier transform of Eq. (3) for periodic boundary conditions is

$$
H_{k}=i d_{0 k} \mathbb{I}+\mathbf{d}_{k} \cdot \boldsymbol{\sigma},
$$

where $\mathbb{I}$ is the $2 \times 2$ identity matrix, $\sigma=\left(\sigma^{x}, \sigma^{y}, \sigma^{z}\right)$ is the vector of Pauli matrices, $\mathbf{d}_{k}=\left(d_{x k}, d_{y k}, d_{z k}\right) \in \mathbb{C}$ is a vector of complex functions of momentum $k$, and $d_{0 k} \in \mathbb{R}$ is a real function of $k$. The energy spectrum is

$$
E_{k}^{ \pm}=i d_{0 k} \pm \sqrt{\mathbf{d}_{1 k}^{2}-\mathbf{d}_{2 k}^{2}+2 i \mathbf{d}_{1 k} \cdot \mathbf{d}_{2 k}}
$$

where $\quad \mathbf{d}_{1 k}=\operatorname{Re}\left[\mathbf{d}_{k}\right], \quad \mathbf{d}_{2 k}=\operatorname{Im}\left[\mathbf{d}_{k}\right] . \quad$ Here, $\quad d_{0 k}=\left(J_{s}-\right.$ $2 \alpha \omega) / 2$ and

$$
\mathbf{d}_{k}=\left(\begin{array}{c}
-J+i \omega G-(\tilde{J}-i \tilde{G} \omega) \cos k \\
-(\tilde{J}-i \tilde{G} \omega) \sin k \\
i \frac{J_{s}}{2}
\end{array}\right)
$$

$E_{k}^{ \pm}$has a real line gap where $\operatorname{Re}\left[E_{k}^{ \pm}\right] \neq 0 \forall k$ provided that $\mathbf{d}_{1 k}^{2}>\mathbf{d}_{2 k}^{2} \forall k$. Systems with a real line gap can have topologically nontrivial phases depending on their symme- try class $[14,15,28]$. Furthermore, since this model includes only short-range hopping, the condition for a topological phase transition can be formulated as in Ref. [28]. We use the symmetry naming conventions of Ref. [14]. We consider four different cases, corresponding to the (i) $\mathcal{P} \mathcal{T}$-symmetric case, (ii), (iii) two different types of breaking of $\mathcal{P} \mathcal{T}$ symmetry, and (iv) a combination of both symmetry-breaking terms.

(i) For $J_{s}=J_{s}^{\mathrm{PT}}=2 \alpha \omega$ and negligible dissipative couplings, $G, \tilde{G}=0, H_{k}$ is $\mathcal{P} \mathcal{T}$ symmetric with $\sigma_{x} H_{k}^{*} \sigma_{x}=H_{k}$. This system is a well-known host of two topologically protected edge modes with $\operatorname{Im} E= \pm 2 \alpha \omega$ for $|J|<|\tilde{J}|$, and it has a real line gap for $|J-\tilde{J}|>\alpha \omega$ [19]. The mode with positive (negative) imaginary energy, i.e., the lasing (lossy) edge mode, corresponds physically to a magnon population that grows (decays) exponentially in time at the left (right) edge. The $\mathcal{P} \mathcal{T}$-symmetric model has generated much interest and has been experimentally realized in photonic systems and microresonators $[14,16]$.

(ii) For $J_{s}=J_{s}^{\mathrm{PT}}$ and $G(\tilde{G}) \neq 0$, Eq. (5) obeys chiralinversion (CI) symmetry $\sigma_{y} H_{k} \sigma_{y}=-H_{-k}$. CI-symmetric models have nontrivial topological phases [20]. The bulk real gap is open for $|J-\tilde{J}|>\omega \sqrt{\delta G^{2}+\alpha^{2}}$, with $\delta G=G-\tilde{G}$, and the condition for two topologically protected edge modes is modified to $|J-i G \omega|<|\tilde{J}-i \tilde{G} \omega|$. 
(iii) Deviations of $J_{s}$ from $J_{s}^{\mathrm{PT}}$ with $G, \tilde{G}=0$ preserve chiral symmetry (CS) defined as $\sigma_{z} H_{k}^{\dagger} \sigma_{z}=-H_{k}$. In this case there is a real gap for $|J-\tilde{J}|>J_{s} / 2$ and the system exhibits a topological phase with two protected edge modes for $|J|<|\tilde{J}|$.

(iv) Including both $G(\tilde{G}) \neq 0$ and $J_{s} \neq J_{s}^{\mathrm{PT}}$ breaks $\mathrm{CI}$ and CS symmetries. The Hamiltonian maintains TRS $^{\dagger}$ symmetry, sometimes called reciprocity [30], defined as $H_{k}^{T}=H_{-k}$. There is a real gap for $|J-\tilde{J}|>\sqrt{\delta G^{2} \omega^{2}+\left(J_{s} / 2\right)^{2}}$; however, $\mathrm{TRS}^{\dagger}$ alone does not guarantee topologically protected phases in 1D [14].

This analysis shows that with dissipative coupling or injected spin current $J_{s} \neq J_{s}^{\mathrm{PT}}$ the model still has a topologically nontrivial phase. Furthermore, only the difference $\delta G$ affects the topological transition and bulk gap closing. Thus, for the parameter regime $G, \tilde{G} \ll \alpha$ and $G \approx \tilde{G}$, in practice resulting from choosing the metallic spacers to be good spin sinks (e.g., Pt), the edge modes are quite robust. Including both $\mathcal{P} \mathcal{T}$-symmetry-breaking terms may compromise the topological protection as $\mathcal{P} \mathcal{T}, \mathrm{CS}$, and CI symmetries are all broken. We find numerically that localized edge modes are still present for $J_{s} \neq J_{s}^{\mathrm{PT}}$ and $G, \tilde{G}=0.1 \alpha$, but these modes may not be topologically protected [14].

The complex frequency spectrum $E$ that results from exact diagonalization of Eqs. (3) and (4) is presented in Fig. 2 for all cases discussed above. For weak dissipative coupling $G, \tilde{G} \ll$ $\alpha$ and $\sim 20 \%$ deviations of $J_{s}$ from the $\mathcal{P} \mathcal{T}$-symmetric value $J_{s}^{\mathrm{PT}}$ we always find a real line gap for $|J| \lesssim|\tilde{J}|$, shown in Figs. 2(a)-2(d), and a lasing edge mode with $\operatorname{Im} E>0$ shown in Figs. 2(e)-2(h). The lasing mode is well separated from the imaginary spectrum of bulk states.

Numerical solutions. To investigate the nonlinear dynamics of the STO array, and, in particular, how nonlinearities affect the growth of the lasing edge modes, we solve Eqs. (1) and (2) numerically. We parametrize the magnetization direction by the power $0<p_{\eta, i}(t)<1$, proportional to the experimentally emitted microwave power, and a precession angle $\phi_{\eta, i}(t)$ as

$$
\mathbf{m}_{\eta, i}=\left(\begin{array}{c}
2 \sqrt{p_{\eta, i}\left(1-p_{\eta, i}\right)} \sin \phi_{\eta, i} \\
2 \sqrt{p_{\eta, i}\left(1-p_{\eta, i}\right)} \cos \phi_{\eta, i} \\
1-2 p_{\eta, i}
\end{array}\right) .
$$

The initial value of $\phi_{\eta, i}(t=0)$ is taken to be a random number between 0 and $2 \pi$ and the initial power $p_{\eta, i}(t=0)$ is drawn from a thermal equilibrium distribution for an ensemble of isolated STOs [21],

$$
P_{\text {eq }} \propto \exp \left[-\frac{N_{s}}{k_{B} T} \omega p_{\eta, i}\right],
$$

where $k_{B}$ is the Boltzmann constant, $T$ the temperature, and $N_{s}$ the number of spins in each STO. We take $2 k_{B} T / N_{s} \hbar \omega=$ $10^{-4}$ and $\alpha=10^{-2}$ for typical materials used for spin-torque experiments at room temperature [21]. For the topologically protected edge modes to occur, the individual oscillators have to be sufficiently phase stable [21], which is the case for these parameters. In Fig. 3 we show the numerical results for the power of all oscillators for $\tilde{J}=-0.025 / 4 \pi \gamma M, \omega / 4 \pi \gamma M=$ $0.5, J / \tilde{J}=0.5$, and $J_{s}=J_{s}^{\mathrm{PT}}$, which corresponds to the $\mathcal{P} \mathcal{T}$ symmetric case supporting one lasing edge mode.

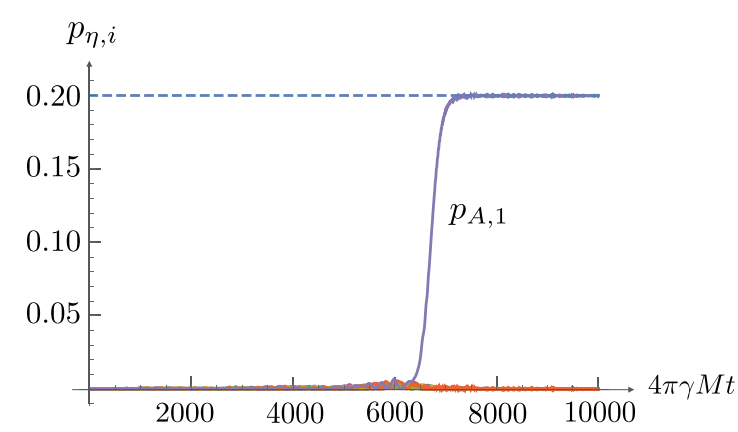

FIG. 3. Power $p_{\eta, i}$ of the oscillator with $\mathbf{m}_{\eta, i}$ in an array of $N=10$ unit cells as a function of time (normalized by the frequency $4 \pi \gamma M)$. The dashed line corresponds to the steady-state power of a single oscillator. Parameters taken are $2 k_{B} T / N_{s} \hbar \omega=10^{-4}$, $\alpha=10^{-2}, \tilde{J}=-0.025 / 4 \pi \gamma M, \omega / 4 \pi \gamma M=0.5, J / \tilde{J}=0.5$, and $J_{s}=J_{s}^{\mathrm{PT}}$.

We find that, after some time, the power of the oscillator on the right edge grows and eventually saturates while the other oscillators have negligible power at all times. The power saturation level of the oscillator at the edge is, because of the small couplings $J$ and $\tilde{J}$, well approximated by the steadystate power of a single oscillator as found from Eq. (1) (see Ref. [21]), denoted by a dashed line in Fig. 3. Our numerical results therefore confirm the presence of a lasing edge mode, which manifests as a topologically protected auto-oscillation of the oscillator at the edge. While we find from our numerical solutions that the dynamics outside the regime of one topologically protected lasing edge mode is interesting, it is also nonuniversal. For example, often several (but not all) oscillators reach a steady state at nonzero power, but which oscillators reach nonzero power strongly depends on the initial conditions. We consider an exhaustive study of the nonlinear dynamics of the proposed system beyond the scope of this Rapid Communication.

Discussion. In this work, we classified the topology of an intrinsically dissipative magnetic system. We establish a mapping between the linearized magnetic dynamics of a 1D array of STOs and a non-Hermitian SSH model. We find that topologically nontrivial phases can be accessed by tuning both the spin-current injection and the properties of the metallic spacers connecting the STOs. The topological phases support a topologically protected lasing edge mode, which manifests as a single edge STO emitting a microwave signal while the bulk STOs are not active. Our results show that the topologically protected edge mode is robust against deviations of the spin current from the $\mathcal{P} \mathcal{T}$-symmetric value and robust against dissipative coupling between STOs.

The emergence and position of the lasing edge mode is controlled via spin-current injection. The location of the lasing edge switches sides upon changing spin-current injection from $A$ to $B$ sublattice sites, thereby opening up prospects for building tunable and robust spin-wave waveguides and neuromorphic networks [31]. Additionally, an extension of our model which exhibits the non-Hermitian skin effect could have applications in sensing [32]. One option in this direction is to consider an electronic net spin current along the direction of the chain that would modify the couplings. Future work could also address the effects of long-range intercell 
and intracell couplings, the effects of periodic driving and coupled dynamics [33], non-Hermitian topology of magnetic systems in higher dimensions $[29,34]$ and topological protection against dipolar interactions or additional spin torques, and phase stability of the lasing edge modes.

Acknowledgments. This research was supported in part by the National Science Foundation under Grant No. NSF
PHY-1748958. H.M.H. acknowledges the support of an NRC Research Assistantship at NIST. This work is supported by the European Research Council via Consolidator Grant No. 725509 SPINBEYOND. R.D. is member of the D-ITP consortium, a program of the Netherlands Organisation for Scientific Research (NWO) that is funded by the Dutch Ministry of Education, Culture and Science (OCW).
[1] K. V. Klitzing, G. Dorda, and M. Pepper, Phys. Rev. Lett. 45, 494 (1980); D. J. Thouless, M. Kohmoto, M. P. Nightingale, and M. den Nijs, ibid. 49, 405 (1982).

[2] C.-X. Liu, S.-C. Zhang, and X.-L. Qi, Annu. Rev. Condens. Matter Phys. 7, 301 (2016).

[3] J. Maciejko, T. L. Hughes, and S.-C. Zhang, Annu. Rev. Condens. Matter Phys. 2, 31 (2011).

[4] B. Yan and C. Felser, Annu. Rev. Condens. Matter Phys. 8, 337 (2017).

[5] M. Sato and Y. Ando, Rep. Prog. Phys. 80, 7 (2017).

[6] J. E. Moore, Nature (London) 464, 194 (2010).

[7] X.-L. Qi, H. Katsura, and A. W. W. Ludwig, Phys. Rev. Lett. 108, 196402 (2012).

[8] P. Liu, J. R. Williams, and J. J. Cha, Nat. Rev. Mater. 4, 479 (2019).

[9] B. K. Stuhl, H. I. Lu, L. M. Aycock, D. Genkina, and I. B. Spielman, Science 349, 1514 (2015); D.-W. Zhang, Y.-Q. Zhu, Y. X. Zhao, H. Yan, and S.-L. Zhu, Adv. Phys. 67, 253 (2019).

[10] Z. Wang, Y. Chong, J. D. Joannopoulos, and M. Soljacic, Nature (London) 461, 772 (2009).

[11] S. D. Huber, Nat. Phys. 12, 621 (2016).

[12] R. Shindou, R. Matsumoto, S. Murakami, and J. I. Ohe, Phys. Rev. B 87, 174427 (2013); R. Chisnell, J. S. Helton, D. E. Freedman, D. K. Singh, R. I. Bewley, D. G. Nocera, and Y. S. Lee, Phys. Rev. Lett. 115, 147201 (2015); S. K. Kim, H. Ochoa, R. Zarzuela, and Y. Tserkovnyak, ibid. 117, 227201 (2016); A. Rückriegel, A. Brataas, and R. A. Duine, Phys. Rev. B 97, 081106(R) (2018); L. Chen, J.-H. Chung, B. Gao, T. Chen, M. B. Stone, A. I. Kolesnikov, Q. Huang, and P. Dai, Phys. Rev. X 8, 041028 (2018).

[13] T. Kikkawa, K. Shen, B. Flebus, R. A. Duine, K. I. Uchida, Z. Qiu, G. E. W. Bauer, and E. Saitoh, Phys. Rev. Lett. 117, 207203 (2016); B. Flebus, K. Shen, T. Kikkawa, K. I. Uchida, Z. Qiu, E. Saitoh, R. A. Duine, and G. E. W. Bauer, Phys. Rev. B 95, 144420 (2017); S. Streib, N. Vidal-Silva, K. Shen, and G. E. W. Bauer, ibid. 99, 184442 (2019).

[14] K. Kawabata, K. Shiozaki, M. Ueda, and M. Sato, Phys. Rev. X 9, 041015 (2019).

[15] Z. Gong, Y. Ashida, K. Kawabata, K. Takasan, S. Higashikawa, and M. Ueda, Phys. Rev. X 8, 031079 (2018); M. A. Bandres and M. Segev, Physics 11, 96 (2018).

[16] S. Weimann, M. Kremer, Y. Plotnik, Y. Lumer, S. Nolte, K. G. Makris, M. Segev, M. C. Rechtsman, and A. Szameit, Nat. Mater. 16, 433 (2017); M. Pan, H. Zhao, P. Miao, S. Longhi, and L. Feng, Nat. Commun. 9, 1308 (2018); M. Parto, S. Wittek,
H. Hodaei, G. Harari, M. A. Bandres, J. Ren, M. C. Rechtsman, M. Segev, D. N. Christodoulides, and M. Khajavikhan, Phys. Rev. Lett. 120, 113901 (2018); R. El-Ganainy, M. Khajavikhan, D. N. Christodoulides, and S. K. Ozdemir, Commun. Phys. 2, 37 (2019); C. Poli, M. Bellec, U. Kuhl, F. Mortessagne, and H. Schomerus, Nat. Commun. 6, 6710 (2015); J. M. Zeuner, M. C. Rechtsman, Y. Plotnik, Y. Lumer, S. Nolte, M. S. Rudner, M. Segev, and A. Szameit, Phys. Rev. Lett. 115, 040402 (2015); L. Xiao, X. Zhan, Z. H. Bian, K. K. Wang, X. Zhang, X. P. Wang, J. Li, K. Mochizuki, D. Kim, N. Kawakami, W. Yi, H. Obuse, B. C. Sanders, and P. Xue, Nat. Phys. 13, 1117 (2017).

[17] T. Chen, R. K. Dumas, A. Eklund, P. K. Muduli, A. Houshang, A. A. Awad, P. Dürrenfeld, B. G. Malm, A. Rusu, and J. Äkerman, Proc. IEEE 104, 10 (2016); J.-V. Kim, Solid State Phys. 63, 217 (2012).

[18] W. P. Su, J. R. Schrieffer, and A. J. Heeger, Phys. Rev. Lett. 42, 1698 (1979).

[19] K. Esaki, M. Sato, K. Hasebe, and M. Kohmoto, Phys. Rev. B 84, 205128 (2011); B. Zhu, R. Lü, and S. Chen, Phys. Rev. A 89, 062102 (2014); S. Lieu, Phys. Rev. B 97, 045106 (2018).

[20] L. Jin and Z. Song, Phys. Rev. B 99, 081103(R) (2019).

[21] A. Slavin and V. Tiberkevich, IEEE Trans. Magn. 45, 1875 (2009).

[22] T. L. Gilbert, IEEE Trans. Magn. 40, 3443 (2004).

[23] J. C. Slonczewski, J. Magn. Magn. Mater. 159, L1 (1996); L. Berger, Phys. Rev. B 54, 9353 (1996).

[24] B. Heinrich, Y. Tserkovnyak, G. Woltersdorf, A. Brataas, R. Urban, and G. E. W. Bauer, Phys. Rev. Lett. 90, 187601 (2003); Y. Tserkovnyak, A. Brataas, and G. E. W. Bauer, Phys. Rev. B 67, 140404(R) (2003).

[25] Y. Tserkovnyak, A. Brataas, G. E. W. Bauer, and B. I. Halperin, Rev. Mod. Phys. 77, 1375 (2005).

[26] R. A. Duine, K.-J. Lee, S. S. P. Parkin, and M. D. Stiles, Nat. Phys. 14, 766 (2018).

[27] T. Holstein and H. Primakoff, Phys. Rev. 58, 1098 (1940).

[28] F. K. Kunst, E. Edvardsson, J. C. Budich, and E. J. Bergholtz, Phys. Rev. Lett. 121, 026808 (2018).

[29] S. Yao and Z. Wang, Phys. Rev. Lett. 121, 086803 (2018).

[30] C. H. Lee and R. Thomale, Phys. Rev. B 99, 201103(R) (2019).

[31] N. Locatelli, V. Cros, and J. Grollier, Nat. Mater. 13, 11 (2014).

[32] J. C. Budich and E. J. Bergholtz, Phys. Rev. Lett. 125, 180403 (2020).

[33] Y. Cao and P. Yan, Phys. Rev. B 99, 214415 (2019).

[34] H. Shen, B. Zhen, and L. Fu, Phys. Rev. Lett. 120, 146402 (2018). 